Received: 5 February 2018

Accepted: 9 July 2018

Published online: 29 August 2018

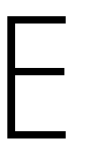

C NTF

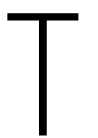

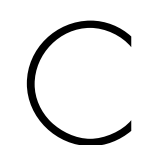

RE
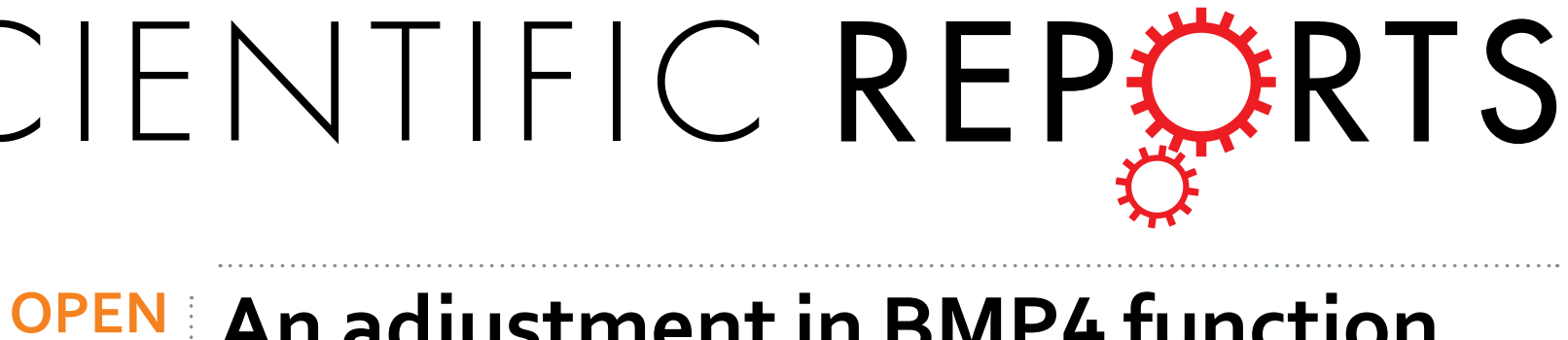

\title{
An adjustment in BMP4 function represents a treatment for diabetic nephropathy and podocyte injury
}

Yui Fujita ${ }^{1}$, Tatsuya Tominaga ${ }^{1}{ }^{1}$, Hideharu Abe ${ }^{1}$, Yumi Kangawa ${ }^{1}$, Naoshi Fukushima ${ }^{2}$, Otoya Ueda ${ }^{2}$, Kou-ichi Jishage ${ }^{2,3}$, Seiji Kishi ${ }^{1}$, Taichi Murakami ${ }^{1}$, Yumiko Saga ${ }^{4}$, Yashpal S. Kanwar ${ }^{5}$, Kojiro Nagai ${ }^{1}$ \& Toshio Doi $^{1}$

Podocyte injury has been proposed to play an important role in diabetic nephropathy; however, its pathological mechanism remains unclear. We have shown that bone morphogenetic protein 4 (BMP4) signaling leads to the glomerular changes characteristic of this disorder. To analyze the molecular mechanism of podocyte injury, the effect of BMP4 was investigated using streptozotocin (STZ)induced, Bmp4 heterozygous knockout (Bmp4+I-) and podocyte-specific Bmp4 knockout mice. Mice with STZ-induced diabetes exhibited glomerular matrix hyperplasia and decreased numbers of podocyte nucleus-specific WT1-positive cells. The number of podocytes and proteinuria were improved in both diabetic Bmp4 knockout mouse models compared to the effects observed in the control mice. The effect of BMP4 overexpression on Bmp4-induced or podocyte-specific transgenic mice was examined. Tamoxifen-induced $B m p 4$-overexpressing mice exhibited mesangial matrix expansion and decreased numbers of WT1-positive cells. Podocyte-specific Bmp4-overexpressing mice displayed increased kidney BMP4 expression and mesangial matrix expansion but decreased nephrin expression and numbers of WT1-positive cells. Both lines of Bmp4-overexpressing mice exhibited increased albuminuria. In cultured podocytes, BMP4 increased phospho-p38 levels. BMP4 decreased nephrin expression but increased cleaved caspase- 3 levels. $\mathrm{p} 38$ suppression inhibited caspase- 3 activation. Apoptosis was confirmed in STZ-diabetic glomeruli and Bmp4-overexpressing mice. Bmp4 $+I-$ mice with diabetes displayed reduced apoptosis. Based on these data, the BMP4 signaling pathway plays important roles in the development of both podocyte injury and mesangial matrix expansion in diabetic nephropathy.

The global prevalence of diabetic nephropathy (DN) has dramatically increased among patients with chronic kidney disease. The associated kidney damage has been shown to be related to functional and structural changes in glomerular cells, including mesangial cells, podocytes, and endothelial cells $s^{1-3}$. Additionally, several recent studies have focused on examining the cellular interactions between different glomerular cells. In particular, mesangial cells are the main target for the development of characteristic mesangial matrix expansion in subjects with $\mathrm{DN}^{4,5}$. As shown in our previous study, bone morphogenetic protein 4 (BMP4)/Smad1 signaling plays an important role in the development of mesangial cell injury in $\mathrm{DN}^{6,7}$. Tamoxifen-inducible $B m p 4$ transgenic mice (tgm), which primarily express BMP4 in glomerular cells, showed dramatic mesangial matrix expansion comparable to that of subjects with DN. However, the involvement of specific cell types and their role(s) in the pathological reaction remain unknown in vivo.

Podocytes are terminally differentiated cells that play an important role in maintaining the barrier function and glomerular structure required to prevent protein leakage into the urine ${ }^{8}$. Proteinuria has been shown to induce glomerular injury by promoting the loss and dysfunction of podocytes ${ }^{9,10}$. Apoptosis constitutes one of

\footnotetext{
${ }^{1}$ Department of Nephrology, Graduate School of Biomedical Science, Tokushima University, Tokushima, Japan. ${ }^{2}$ Research Division, Fuji Gotemba Research Labs, Chugai Pharmaceutical Co., Ltd, Shizuoka, Japan. ${ }^{3}$ Chugai Research Institute for Medical Science Inc., Shizuoka, Japan. ${ }^{4}$ Division of Mammalian Development, Genetic Strains Research Center, National Institute of Genetics, Mishima, Shizuoka, Japan. ${ }^{5}$ Department of Pathology \& Medicine-Nephrology, FSM, Northwestern University, Chicago, Illinois 60611, USA. Yui Fujita and Tatsuya Tominaga contributed equally. Correspondence and requests for materials should be addressed to T.T. (email: tominaga. tatsuya@tokushima-u.ac.jp)
} 
the main pathways contributing to podocyte loss, which leads to glomerulosclerosis ${ }^{11}$. Podocytes are subjected to various stresses, including oxidative stress, endoplasmic reticulum stress, metabolic stress, inflammation, toxins, and physical-mechanical stimuli ${ }^{12,13}$. These factors may be involved in the complex mechanism underlying the development of kidney damage. For example, high glucose and advanced glycated proteins induce podocyte apoptosis, a process mediated by transforming growth factor beta (TGF $\beta$ ), in conjunction with p $38^{14}$. Specifically, the activation of the p38 MAP kinase and caspase- 3 in cells exposed to stress or cytokines triggers the apoptotic response in podocytes.

BMPs are expressed during kidney development and affect tissue morphogenesis. BMP4 is an important molecule that induces the stem cell differentiation required for the development of various organs, including the kidney ${ }^{15-17}$. BMP4 also induces Smad1/5/8 phosphorylation and p38 activation during embryonic tissue formation, triggering apoptosis ${ }^{18}$. Additionally, the BMP4-Smad1 pathway mediates senescence in injured cells ${ }^{19}$. Consistent with podocyte injury in nephrotic syndrome, which is caused by apoptosis and cell cycle dysfunction, we have reported that BMP4 constitutes a critical molecule that induces glomerulosclerosis in subjects with DN. In addition, the expression of p16 and p21 in response to BMP 4 has been shown to be mediated by the Smad1/5/8 signaling pathway ${ }^{20}$. Smad1 upregulates the transcription of type IV collagen, the primary protein component of the extracellular matrix in mesangial cells ${ }^{6}$. Notably, mesangial matrix hyperplasia in DN mice is suppressed by a BMP4 neutralizing antibody ${ }^{21}$. Thus, BMP4/Smad1 signaling plays a central role in the development of mesangial matrix hyperplasia in subjects with $\mathrm{DN}$; however, the precise molecular mechanisms by which BMP4 induces podocyte injury remain unclear. In this study, we used Bmp4 heterozygous knockout mice $(B m p 4+/-)$, podocyte-specific Bmp 4 knockout mice, induced Bmp4 tgm, and podocyte-specific Bmp4 tgm to examine the molecular mechanism by which the BMP4/Smad1/p38 signaling pathway mediates glomerular injury in a mouse model of DN.

\section{Results}

Diabetic Bmp4 heterozygous knockout mice exhibit reduced podocyte injury. We first examined the development of DN in STZ-induced diabetes models using Bmp4+/-mice. High blood glucose levels were observed at 24 weeks after the induction of diabetes in either wild-type or Bmp4+/-mice, as we have previously reported $^{7}$. Glycoalbumin levels were also significantly increased in the diabetic group (Supplemental Table 1A). A significant difference in blood glucose levels was not observed between the wild-type and Bmp $4+/-$ mice. The diabetic mice exhibited significantly increased mesangial matrix expansion and BMP4 levels compared to those in the control mice (Fig. 1C-a and c). The area of BMP4 expression partially overlapped podocytes, but BMP4 expression was also observed elsewhere, including primarily mesangial areas (Fig. 1A-b).

Next, we investigated whether a decrease in BMP4 expression attenuated any renal changes in diabetic mice. Mesangial matrix expansion, BMP4 expression, and albuminuria were attenuated in the diabetic Bmp $4+/-$ mice compared with the diabetic wild-type mice (Fig. 1B,C-g-h and D). The results of periodic acid methenamine silver (PAM)-stained areas in the wild-type mice were compatible with findings from our previous report ${ }^{7}$. A statistically significant difference in the albuminuria levels was not observed between the diabetic and non-diabetic Bmp4 +/-mice. The podocyte number, as determined by the number of WT1-positive cells, was maintained in the diabetic Bmp4 $+/$ - mice compared to the diabetic wild-type mice (Fig. 1C-k,l and E). Furthermore, the diabetic Bmp4+/-mice expressed higher levels of nephrin than the diabetic wild-type mice (Fig. 1C-o,p and F). BMP4-positive staining was only observed in the wild-type mice, and the area was increased in the diabetic animals (Fig. 1G). Western blot analyses revealed decreased expression of podocin in the diabetic mice. The diabetic $B m p 4+/-$ mice exhibited similar levels of podocin as the nondiabetic mice (Fig. $1 \mathrm{H}$ ). In addition, the creatinine clearance rate (Ccr) was slightly reduced in the diabetic Bmp4 $+/-$ mice (Supplemental Table 1A).

Podocyte-specific Bmp4 knockout mice with diabetes exhibit reduced podocyte injury. We investigated whether the podocyte-specific reduction in levels of secreted BMP4 attenuated any renal changes in diabetic mice. $B m p 4^{\text {loxP }} \times$ Podocin-Cre mice exhibited decreased Bmp4 levels in the kidneys, as detected by quantitative PCR. Bmp $4^{l o x P}$ induction (-) in control mice has not been crossbred with Podocin-Cre mice. Mesangial matrix expansion was attenuated in the diabetic $B m p 4^{\text {loxP }} \times$ Pod-Cre mice compared with the diabetic Bmp $4^{\text {loxP }}$ induced (-) mice (Fig. 2A-c-d and B). The reduction in WT1- and nephrin-positive areas was alleviated in these mice compared to the diabetic non-induced mice (Fig. 2 A-k-l,o-p,C,and D). Electronic microscopy analyses of diabetic $B m p 4^{l o x P} \times$ Pod-Cre mice showed normal podocyte foot processes and glomerular basement membranes (GBMs) (Fig. 2A-t). Diabetic Bmp $4^{\text {loxP }}$ induced (-) mice displayed marked alterations such as GBM thickening and podocyte foot process effacement (Fig. 2A-s). In addition, Ccr and albuminuria were not different between diabetic $B m p 4^{l o x P} \times$ Pod-Cre mice and non-diabetic mice (Supplemental Table 1B).

Bmp4 overexpression induces podocyte injury and mesangial matrix expansion in vivo. To investigate the role of BMP4 in adult mice, we generated Bmp4 tgm using the tamoxifen-regulated Cre-loxP system. As previously reported (ref. ${ }^{7}$ ). The Bmp4 tgm (+) is a tamoxifen-induced BMP4-expressing adult mouse strain. Bmp4 tgm (-) control mice were not induced with tamoxifen. Bmp4 tgm (+) displayed increased BMP4 expression in whole glomeruli, as shown in red (Fig. 3A-d) and glomerulosclerosis (Fig. 3A-b). These mice exhibited a significant decrease in the number of WT1-positive cells and nephrin-positive area compared to Bmp 4 tgm $(-)$ control mice (Fig. 3A-f and h). Bmp4 tgm (+) exhibited a decreased number of WT1-positive cells compared with Bmp4 tgm (-) (Fig. 3B). We confirmed the podocin levels using western blotting in vivo. Bmp4 tgm (+) displayed decreased podocin levels compared with those in Bmp4 tgm (-) (Fig. 3C).

Podocyte-specific Bmp4 transgenic mice display podocyte loss. CAG-B4 $\times$ Pod-Cre mice exhibited increased BMP4 expression in the kidney, as detected by western blotting and quantitative PCR. The CAG-B4 
A

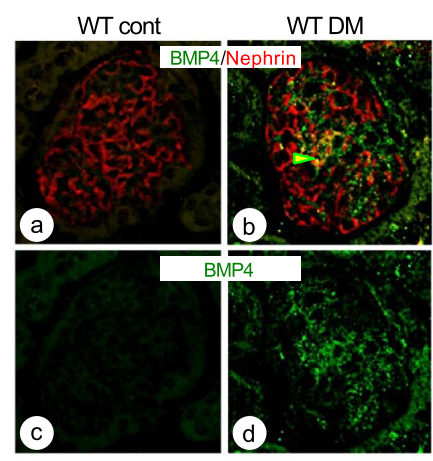

B

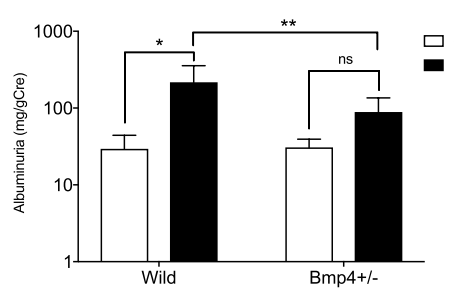

D

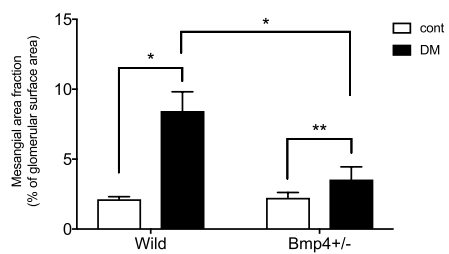

G

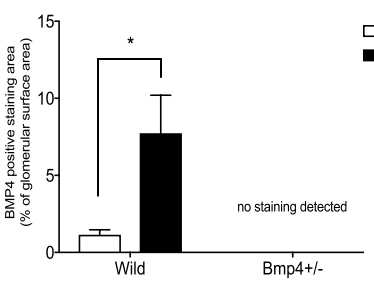

C

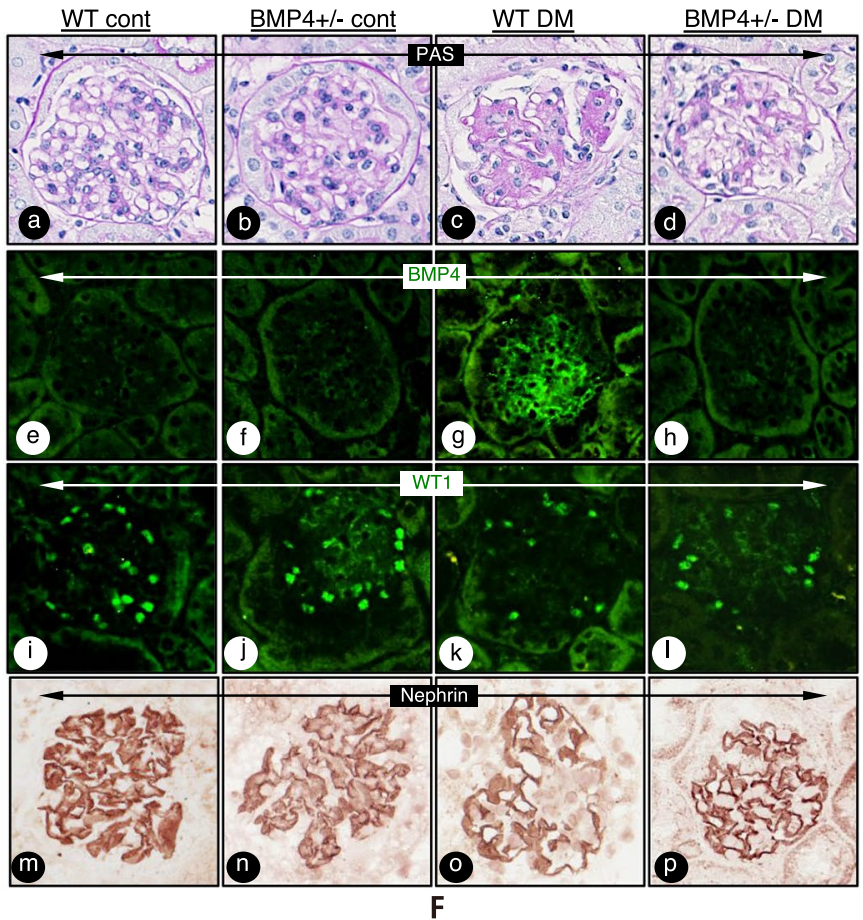

$\mathrm{E}$
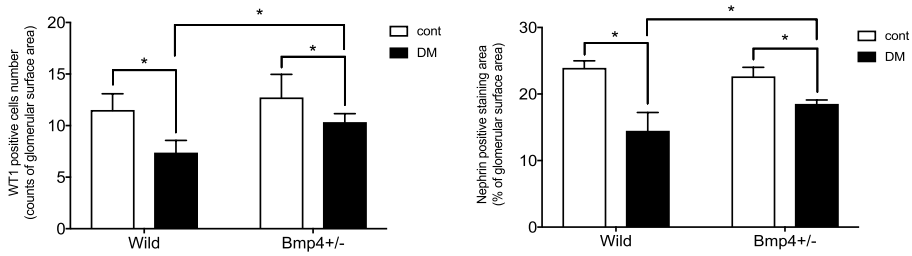

H

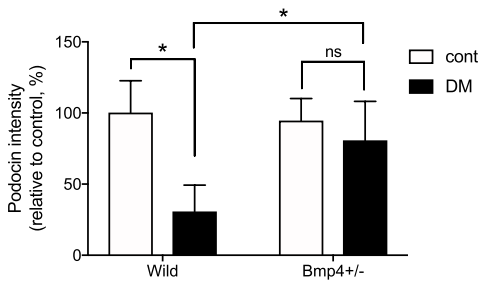

Figure 1. Diabetic Bmp4 heterozygous knockout mice (Bmp4+/-) exhibited reduced podocyte injury and mesangial matrix expansion. (A) Images of BMP4 and nephrin immunohistochemistry in the glomeruli. In diabetic C57BL/6 mice (WT DM) (b), BMP4 expression was increased in the glomerulus compared with the control mice (WT cont) (a). The BMP4-expressing areas are depicted in green, and the nephrin-expressing area is shown in red. Yellow indicates the area of BMP4 and nephrin overlap (b, arrowhead). (B) Albuminuria was $214.7 \mathrm{mg} / \mathrm{g}$ Cre for WT DM mice and $87.9 \mathrm{mg} / \mathrm{g}$ Cre for $B m p 4+/-$ DM mice. A significant difference was not observed between Bmp4 +/ - cont and Bmp4 +/- DM mice. (C) Representative images of light microscopy and immunohistochemistry in the glomeruli. WT DM mice exhibited increased BMP4 expression (g) and decreased numbers of WT1-positive cells (k) and nephrin-expressing areas (o) compared with the WT controls. $B m p 4+/-D M$ mice did not display BMP4-positive staining (h). Bmp4 +/-DM mice exhibited a moderate decrease in the number of WT1-positive cells (l) and nephrin expression (p) compared with WT DM mice. (D) The mesangial area fraction was determined by measuring the PAM-stained area. A significant difference was not observed between WT cont and $B m p 4+/-$ cont mice. WT DM displayed mesangial matrix expansion compared with the control mice. Bmp4+/-DM mice exhibited decreased mesangial matrix expansion compared with WT DM mice. (E) The number of WT1-positive cells was determined by immunostaining. A greater number of WT1-positive cells was observed in Bmp4+1-DM mice than in WT DM mice. (F) The nephrin-positive area was determined by measuring the immunostaining. A larger nephrin-positive area was observed in Bmp4+/-DM mice than in WT DM mice. (G) The BMP4-positive area was determined by measuring the immunostaining. WT DM mice exhibited increased BMP4 expression. $(\mathbf{H})$ Podocin levels were determined by western blotting. Levels in WT DM mice were $30.5 \%$ and levels in $B m p 4+1-\mathrm{DM}$ mice were $80.7 \%$ compared to WT cont mice. Cont, nondiabetic mice; DM, diabetic mice. ${ }^{*} P<0.01,{ }^{* *} P<0.05$ (ANOVA). 
A

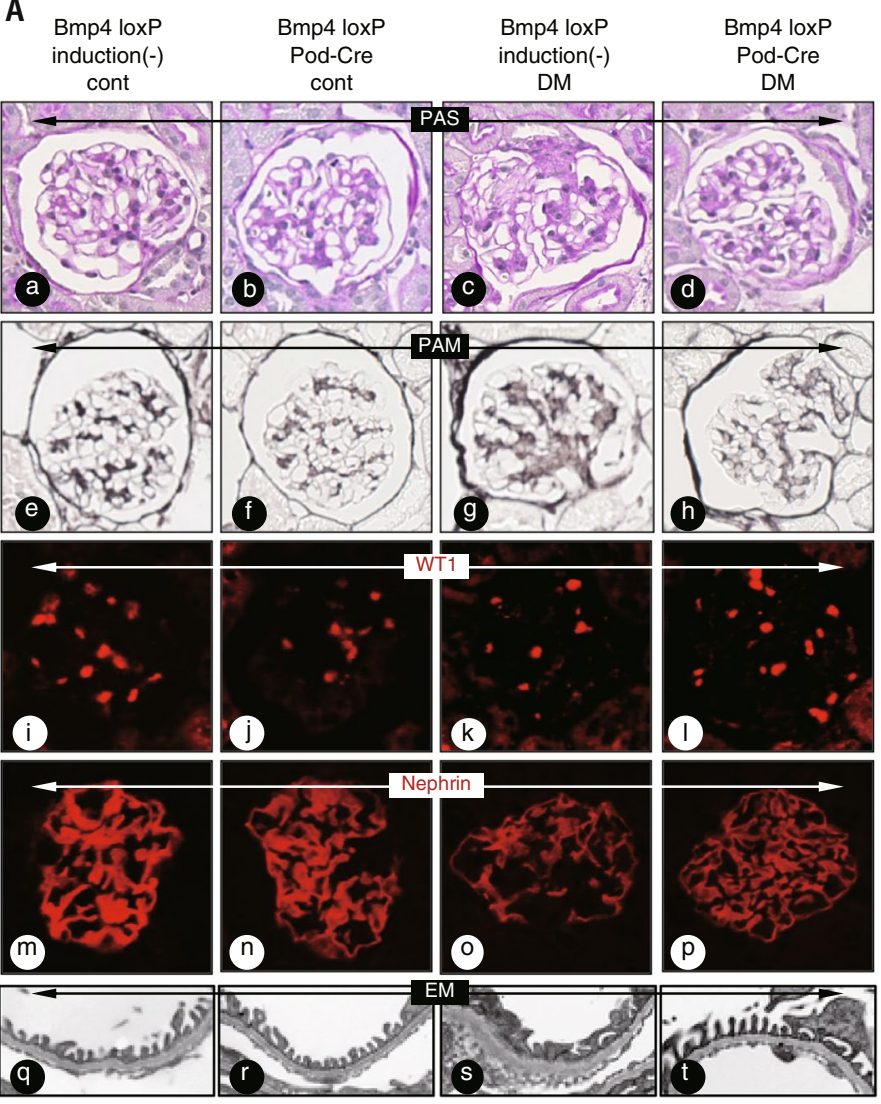

B

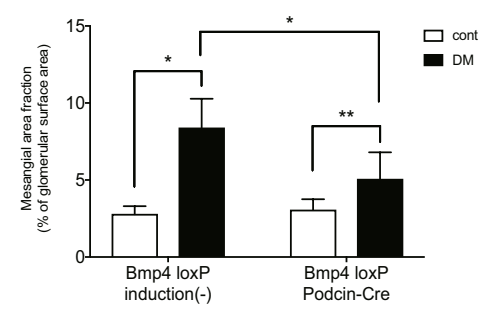

C

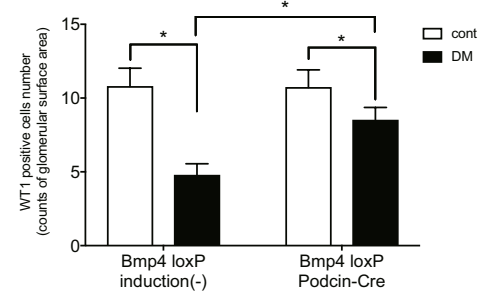

D

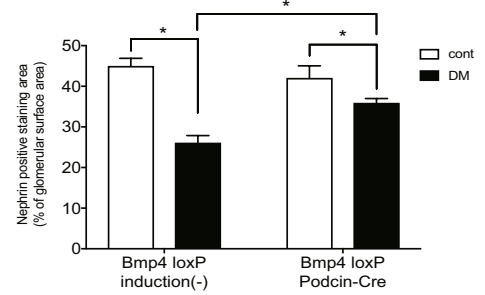

Figure 2. Diabetic podocyte-specific Bmp4 knockout mice exhibited reduced podocyte injury and mesangial expansion. (A) Representative light microscopy, immunohistochemistry and electron microscopy images of glomeruli. Extracellular matrix deposition was determined by PAS staining. PAS staining was increased in diabetic $B m p 4^{l o x P}$ induced (-) mice (c) and decreased in diabetic $B m p 4^{\text {loxP }} \times$ Podocin-Cre mice (d). Diabetic $B m p 4^{\text {loxP }} \times$ Podocin-Cre mice exhibited a moderate decrease in the number of WT1-positive cells (l) and nephrin expression (p) compared with diabetic Bmp $4^{\text {loxP }}$ induced (-) mice. The GBM thickness in the diabetic $B m p 4^{\text {loxP }} \times$ Podocin-Cre mice was reduced compared with the diabetic $B m p 4^{\text {loxP }}$ induced $(-)$ mice $(\mathrm{s}$ and $\mathrm{t})$. (B) A bar graph summarizes the histological scores for PAM staining. Diabetic Bmp $4^{\text {loxP }} \times$ Podocin-Cre mice displayed decreased mesangial matrix expansion compared with diabetic $B m p 4^{l o x P}$ induced (-) mice. (C) The number of WT1-positive cells was determined by immunostaining. A greater number of WT1-positive cells was observed in the diabetic Bmp $4^{\operatorname{lox} P} \times$ Podocin-Cre mice than in the diabetic Bmp $4^{\operatorname{lox} P}$ induced (-) mice. (D) The nephrin-positive area was determined by measuring the immunostaining. A larger nephrin-positive area was observed in the diabetic $B m p 4^{\text {loxP }} \times$ Podocin-Cre mice than in the diabetic $B m p 4^{\text {loxP }}$ induced $(-)$ mice. Cont, nondiabetic mice; DM, diabetic mice. ${ }^{*} P<0.01,{ }^{* *} P<0.05$ (ANOVA).

control mice were not crossbred with Podocin-Cre mice. CAG-B4 $\times$ Pod-Cre mice showed mesangial matrix hyperplasia at 30 weeks, and progressive mesangial matrix hyperplasia at 40 weeks of age (Fig. $4 \mathrm{G}-\mathrm{g}$ and $\mathrm{h}$ ). The 60 -week-old mice developed severe mesangial sclerosis accompanied by interstitial fibrosis. In particular, the CAG-B4 $\times$ Pod-Cre mice showed increased BMP4 expression in the glomerulus. These BMP4-expressing areas colocalized with podocytes, although staining was also observed in the area expressing collagen IV (Fig. 4E-b). These mice displayed fewer podocytes at 40 weeks of age than the reference mice (Fig. 4C), and they also exhibited significantly reduced nephrin expression (Fig. 4D). Furthermore, an increased number of pSmad1-positive cells was observed not only in partially WT1-positive cell areas but also among areas of predominantly mesangial cells (Fig. 4A-1). The electron microscopy analyses of these mice revealed GBM thickening, podocyte foot process effacement, and mesangial expansion, similar to the findings of DN mice (Fig. $4 \mathrm{~A}-\mathrm{n}$ ). The CAG-B4 $\times$ Pod-Cre mice exhibited decreased podocin levels compared with those in the $C A G-B 4$ control mice (Fig. $4 \mathrm{~F}$ ). In addi-

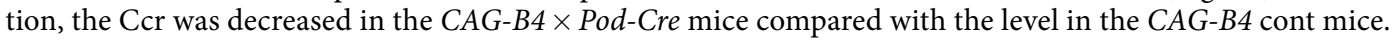
Albuminuria was increased in the $C A G-B 4 \times$ Pod-Cre mice compared with the level in the $C A G-B 4$ control mice.

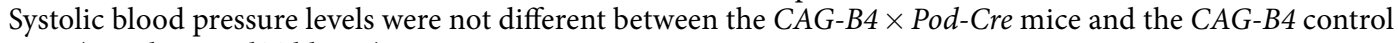
mice (Supplemental Table 1D).

Podocyte apoptosis is induced by the BMP4/p38 pathway. We studied cultured podocytes in vitro to investigate the mechanism by which BMP4 mediates podocyte injury. Podocytes were treated with BMP4 for $5 \mathrm{~min}$ to $24 \mathrm{~h}$. Western blot analyses revealed a time-dependent increase in phosphorylated p38 levels following 
A

BMP4 Tgm(-) BMP4 Tgm(+)
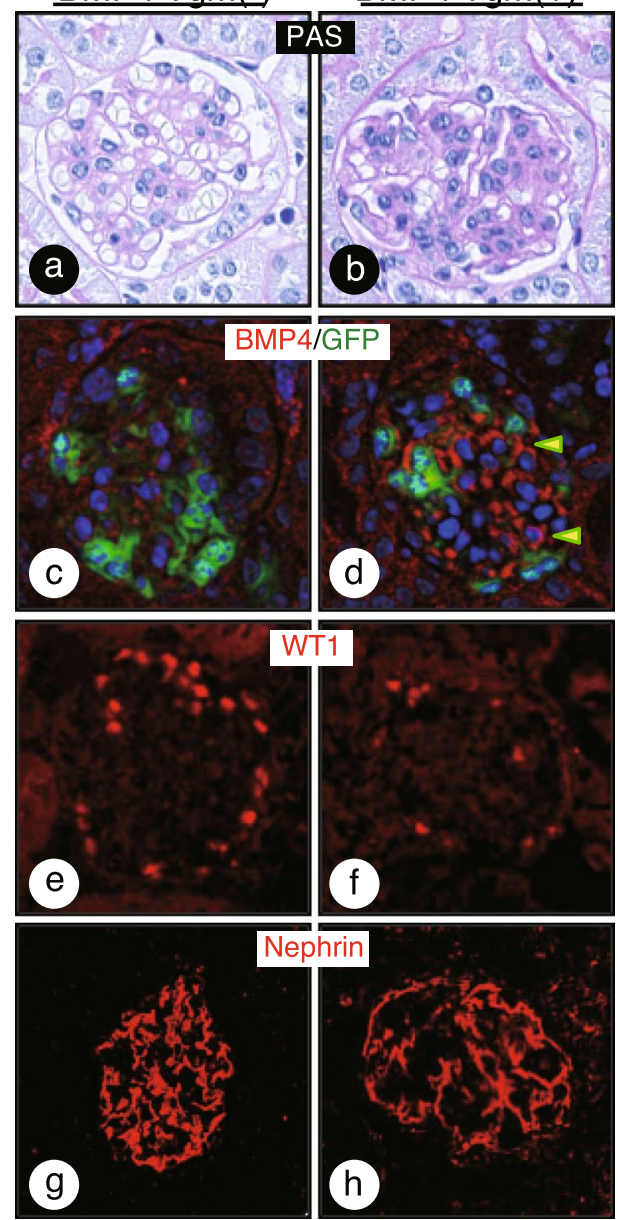

B

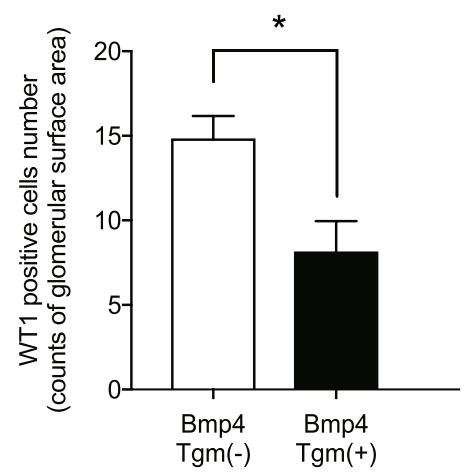

C

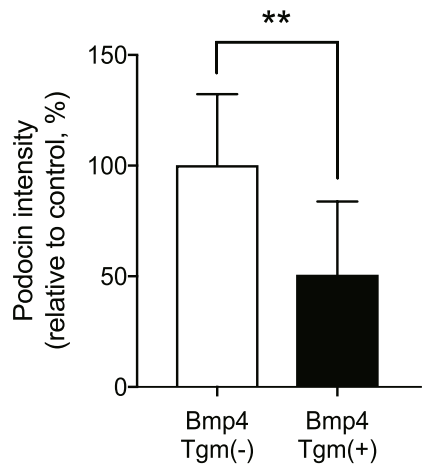

Figure 3. Tamoxifen-induced Bmp4 tgm exhibited podocyte injury and mesangial matrix expansion. (A) Representative light microscopy and immunohistochemistry images of glomeruli. Tamoxifen-induced expression of BMP4 in the transgenic mice resulted in the expansion of the mesangial area (b). Tamoxifen administration reduced GFP fluorescence and increased levels of the BMP4 protein. BMP4 was located in cells that previously expressed GFP (d). Compared to untreated animals, the WT1- (f) and nephrin-positive areas (h) were reduced in the tamoxifen-treated BMP4 tgm. (B) The number of WT1-positive cells was determined by immunostaining. Fewer WT1-positive cells were observed in Bmp4 tgm (+) than in Bmp4 tgm (-). (C) Podocin levels were determined by western blotting. Podocin levels were decreased in $B m p 4$ tgm $(+)$ compared with $B m p 4 \operatorname{tgm}(-) .{ }^{*} P<0.01,{ }^{* *} P<0.05$ (t-test).

treatment with BMP4 (Fig. 5A). In contrast, nephrin expression was reduced by BMP4 (Fig. 5B). The BMP4 treatment induced caspase-3 cleavage in podocytes (Fig. 5B). P38 inhibitors (SB242235 and SB202190) inhibited the activation of $\mathrm{p} 38$ and cleaved caspase-3 (Fig. 5C). Conversely, dorsomorphin specifically inhibited the activation of Smad1, whereas cleaved caspase-3 levels were not affected (Fig. 5C).

Changes in the levels of cleaved caspase-3 and apoptosis in vivo. As these findings suggest that $\mathrm{BMP} 4 / \mathrm{p} 38 /$ caspase-3 signaling may play a key role in establishing podocyte injury, we investigated the involvement of these molecules in vivo. Cleaved caspase-3 levels were increased in the glomeruli of STZ-induced DN mice (Fig. 6A-c). The area expressing cleaved caspase-3 overlapped with both mesangial cells and podocytes. STZ-induced Bmp4 knockout mice exhibited reduced levels of cleaved caspase-3 (Fig. 6A-c and d), whereas increased levels of cleaved caspase- 3 were detected in the tamoxifen-induced Bmp 4 and CAG-B4 $\times$ Pod-Cre mice (Fig. 6A-f and h). Diabetic-induced nuclear damage in wild-type mice was reflected by increased apoptosis, as highlighted by TUNEL staining. The diabetic $B m p 4+/-$ mice exhibited notably reduced apoptotic changes, as assessed by TUNEL staining (Fig. 6B-c and d). Bmp4 tgm (+) and CAG-B4 $\times$ Pod-Cre mice displayed nuclear damage due to apoptosis (Fig. 6B-j red color and l green color). The green color in images of Bmp4 tgm is GFP, which is derived from the recombinant gene. 
A
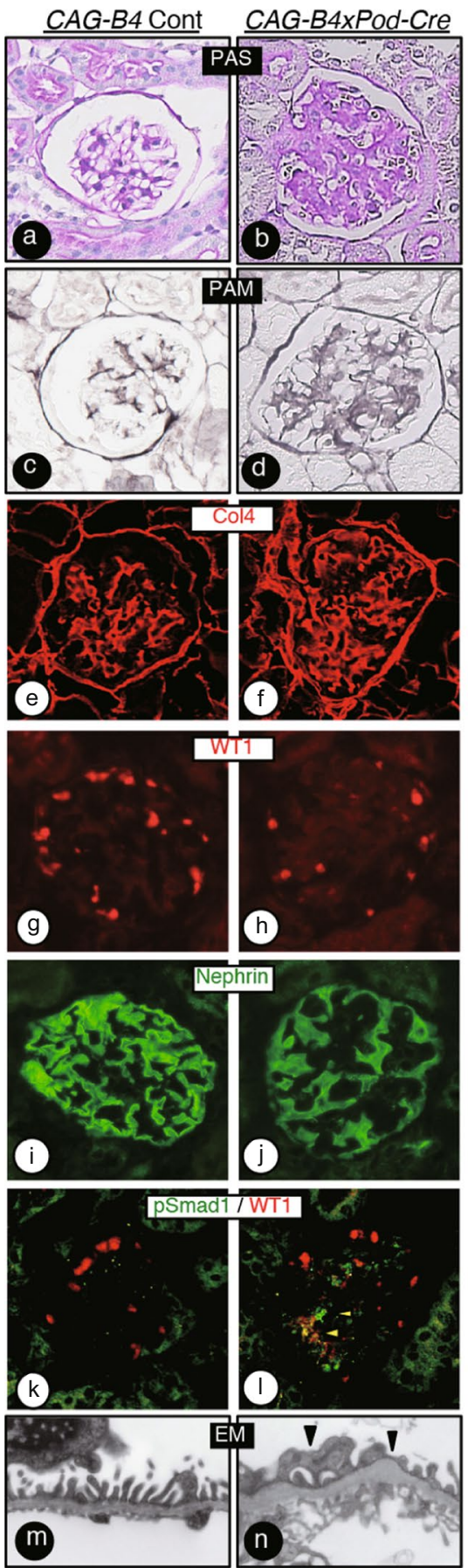

B

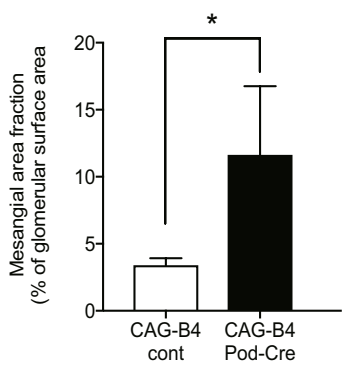

C

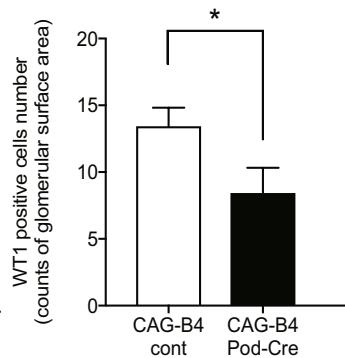

D

E

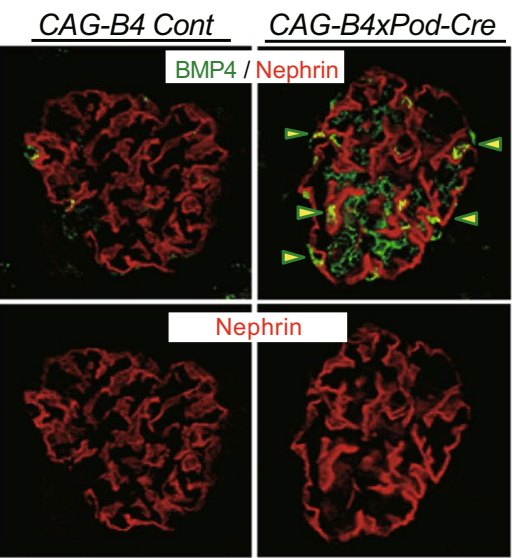

$\mathbf{F}$

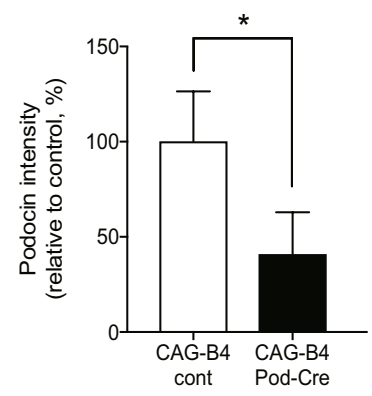

G

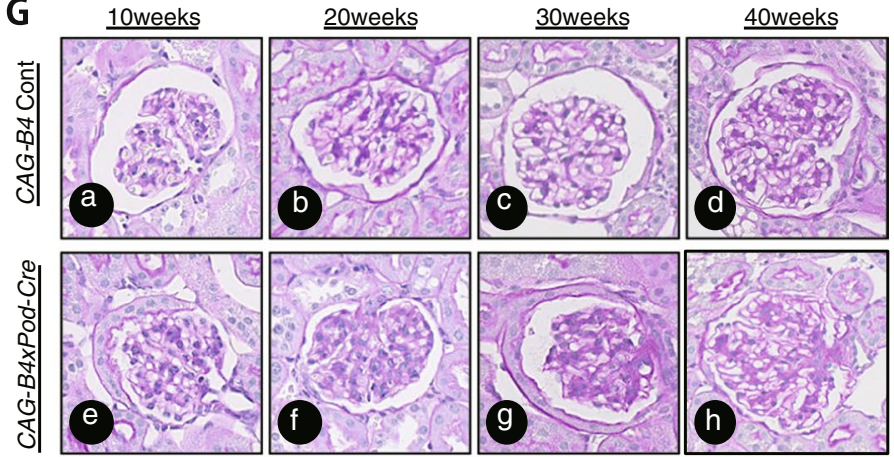

Figure 4. Podocin-Cre Bmp4 tgm exhibited glomerular injury. (A) Representative light microscopy, immunohistochemistry and electron microscopy images of glomeruli. The left panel shows a non-induced mouse (CAG-Bmp4 cont), and the right panel shows a Podocin-Cre induced Bmp4tgm (CAG-B4xPod-Cre). Extracellular matrix deposition was determined by PAS staining. PAS staining was increased in $C A G-B 4 x P o d-$ Cre mice (b). The Col4-expressing area was increased in CAG-B4xPod-Cre mice (f). Compared to the CAG$B m p 4$ cont mice, the WT1 - (h) and nephrin-positive areas (j) were reduced in CAG-B4xPod-Cre mice. The number of phospho-Smad1 (pSmad1)-positive cells was increased in CAG-B4xPod-Cre mice compared with $C A G-B m p 4$ cont mice (l). The GBM thickness was increased in CAG-B4xPod-Cre mice compared with CAG$B m p 4$ cont mice ( $\mathrm{m}$ and $\mathrm{n}$ ). (B) A bar graph summarizes the histological scores of PAM staining. CAG-B4xPodCre mice exhibited mesangial matrix expansion compared with $C A G-B m p 4$ cont mice. (C) The number of WT1-positive cells was obtained by immunostaining. Fewer WT1-positive cells were observed in CAG-B4xPodCre mice than in CAG-Bmp4 cont mice. (D) The nephrin-positive area was determined by measuring the immunostaining. The nephrin-positive area was decreased in CAG-B4xPod-Cre mice compared with CAG-Bmp4 cont mice. (E) Images of BMP4 and nephrin immunohistochemical staining in glomeruli. In CAG-B4xPod-Cre mice, BMP4 expression was increased in the glomerulus compared with that in $C A G-B m p 4$ cont mice. 
The BMP4-expressing areas are shown in green, and the nephrin area is shown in red. Yellow indicates the area of BMP4 and nephrin overlap (arrowhead). (F) Podocin levels were determined by western blotting. Podocin levels were decreased in CAG-B4xPod-Cre mice compared with those in CAG-Bmp4 cont mice. G) Glomerular PAS staining in each mouse from 10 weeks to 40 weeks of age. PAS staining was increased in CAG-B4xPod-Cre mice aged more than 30 weeks (g and $\mathrm{h}$ ). ${ }^{*} \mathrm{P}<0.01$, (t-test).

\section{Discussion}

Podocyte injury and loss have been indicated as the crucial markers of the pathogenesis of glomerular injury; however, the detailed molecular mechanisms and cell-to-cell responses remain to be elucidated, because multiple factors may cause podocyte injury. In the glomerulus, three types of cells (i.e., endothelial, mesangial, and parietal epithelial) respond to podocyte injury. Endothelial and mesangial cells are connected to podocyte cells across the glomerular basement membrane. However, the detailed mechanisms underlying the relation between the mesangium and podocyte injury are unclear. DN is characterized by mesangial matrix expansion, which is caused by the excess deposition of extracellular matrix proteins in the mesangial area, as detected by increased expression of type IV collagen. The initial finding of the present study was that BMP4 secreted by glomerular cells, including mesangial cells and podocytes, directly caused podocyte injury and correlated with urinary albumin excretion. BMP4 secreted from cells adheres to collagen IV ${ }^{22}$. We postulate that BMP4 secreted from podocytes accumulates in glomerular mesangial cells, in which collagen IV is a primary component. Concomitantly, BMP4 accumulates in mesangial cells, activates Smad1, and increases the production of extracellular matrix proteins in mesangial cells. BMP4 expression was primarily observed in mesangial cells, as well as in podocytes. BMP4 is a secreted protein that may act on various cells in a paracrine manner. Therefore, BMP4 signaling pathways play central roles in the development of podocyte injury and mesangial matrix expansion in subjects with DN.

P38 MAPK signaling mediates BMP4-induced apoptosis and senescence in cancer cells ${ }^{23}$, whereas BMP4-induced Smad1 signaling activates apoptotic cell death in embryonic stem cells ${ }^{24}$. Although the p38 MAPK and Smad1 pathways have been reported to synergistically induce apoptosis and autophagy in tumor cells ${ }^{25}$, other studies have reported that the p38 MAPK pathway is separate from Smad1 signaling because it mediates NRAGE-p38 signaling ${ }^{26}$. In particular, the mechanism underlying podocyte damage depends on BMP4/BMP receptor signaling in the early stage of diabetes. BMP4 induced podocyte apoptosis mediated by p38 activation and cleaved caspase-3 (Fig. 5B). This pathway induced podocyte loss, as detected by the number of WT1-positive cells, and subsequent foot process injury, as evidenced by nephrin staining (Fig. 4A-h,j and n).

R-Smad has been reported to affect the cell cycle; accordingly, Smad1 may impact senescence in podocytes ${ }^{27}$. Alternatively, we considered that Smad1 upregulated the extracellular matrix expansion in mesangial cells, based on our observation that phosphorylated Smad1 directly induces collagen IV expression in mesangial cells ${ }^{6}$. The Smad1 inhibitor did not affect the levels of cleaved caspase- 3 in cultured podocytes. However, the induction of apoptosis was suppressed by p38 inhibitors (Fig. 5C). BMP4 exerts two direct effects on glomeruli injury, including the effect of BMP4/Smad1 signaling on mesangial matrix expansion and the effect of BMP4/p38 signaling on podocyte injury. As shown in our previous study, a BMP4 neutralizing antibody blocked Smad1-mediated mesangial matrix expansion ${ }^{21}$. Based on these findings, BMP4 signaling represents a promising target for developing a therapeutic approach for DN.

Podocyte-specific Bmp4 tgm showed typical mesangial sclerosis accompanied by podocyte loss and increased phosphorylated Smad1 levels in mesangial cells. Thus, podocyte loss itself is responsible for mesangial sclerosis, and the increased BMP4 expression observed in podocytes induces Smad1 activation in mesangial cells in a paracrine fashion. Based on these findings, BMP4/p38 signaling in podocytes and BMP4/Smad1 signaling in mesangial cells are potential therapeutic targets for DN. Because BMP4 regulates both podocyte loss and mesangial Smad1 activation in DN, BMP4 represents an essential molecule involved in the development of the typical pathological features of DN.

Both podocyte loss and mesangial expansion were concomitantly observed in most pathological cases of DN; however, microalbuminuria did not completely reflect the characteristic renal pathological changes, including mesangial matrix expansion and glomerular basement membrane thickening, in the early stages of human DN. Urinary albumin excretion is affected by the charge and size of the barrier of the glomerular basement membrane $^{28,29}$. The mechanism of renal disease progression requires a subsequent in-depth analysis of the relationship between podocyte dysfunction and GBM disruption. Bmp4 knockout mice exhibited reduced podocyte injury and albuminuria levels. These initial observations provide sufficient evidence that BMP4 is a therapeutic target. Based on the findings of the present study, we speculate that BMP4 induces podocyte injury in an early stage of $\mathrm{DN}$ in which microalbuminuria is observed. Additional studies are necessary to confirm these observations.

\section{Experimental Procedures}

Experimental animals. All animal experiments were performed in accordance with the guidelines of the Institutional Animal Care and Use Committee of Chugai Pharmaceutical Co., Ltd. and the Tokushima University animal facility, and the Ethical Review Board of Tokushima University granted permission for the procedures performed in this study.

Bmp4-knockout mice (C57BL/6 Bmp4+/-) and conditional knockout mice (C57BL/6 Bmp $4^{\text {loxP }}$ ) were gifts from B. Hogan and M. Saitou. The generation and characterization of these mutant mice have been described elsewhere ${ }^{16,30}$. The Bmp4 $+/$ - mice were maintained on a C57BL/6 background. The Podocin-Cre (Pod-Cre) mice were obtained from The Jackson Laboratory (Bar Harbor, ME, USA). We crossed Pod-Cre mice with Bmp $4^{\text {loxP }}$ mice to generate the podocyte-specific Bmp4 knockout mice. Diabetes was induced in 8-week-old male C57BL/6 mice weighing $22-24 \mathrm{~g}$ by the intraperitoneal injection of $50 \mathrm{mg}$ per $\mathrm{kg}$ body weight streptozotocin (STZ) in 
A

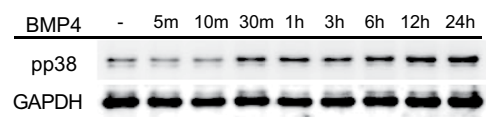

B

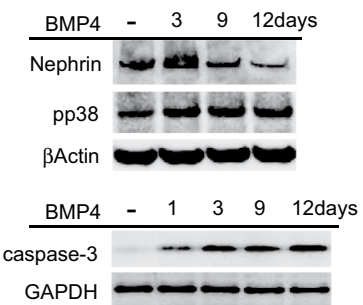

C

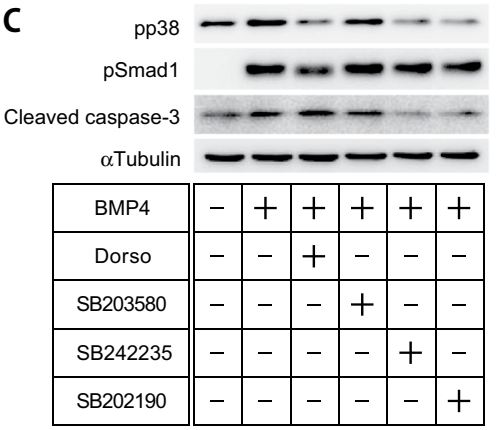

Figure 5. BMP4 induced apoptotic signaling in cultured podocytes. (A) Cultured podocytes were treated with BMP4 $(20 \mathrm{ng} / \mathrm{ml})$ at the indicated time points. BMP4 treatment of podocytes increased levels of phosphorylated p38 in a time-dependent manner. (B) BMP4 decreased nephrin expression and increased cleaved caspase-3 levels after 12 days of stimulation. (C) Cultured podocytes were untreated or incubated with dorsomorphin (Dorso), SB203580, SB242235 or SB202190 for $24 \mathrm{hr}$ and then stimulated with BMP4. Each inhibitor was added to the culture medium at a concentration of $500 \mathrm{nM}$. The pp38 levels were decreased in cells treated with dorsomorphin, SB242235 and SB202190. The pSmad1 levels were decreased in response to dorsomorphin treatments. The cleaved caspase-3 levels were decreased in cells treated with SB242235 and SB202190. Total cell lysates were immunoblotted with anti-pp38, anti-nephrin, anti-cleaved caspase-3, anti-pSmad1, anti-GAPDH, anti- $\beta$-actin and anti- $\alpha$-tubulin antibodies.

citrate buffer, $\mathrm{pH} 4.5$, for 5 consecutive days. The diabetic state was confirmed 4 weeks after the final injection by measuring the blood glucose levels with an Accu-Chek monitor (Roche). All mice that were administered STZ had a blood glucose concentration exceeding $400 \mathrm{mg} / \mathrm{dl}$ and were considered diabetic. Reference mice were injected with citrate buffer. The mice were classified into four groups: $(\mathrm{A})$ nondiabetic C57BL/6 $(\mathrm{n}=7)$, (B) nondiabetic Bmp4 $+/-(\mathrm{n}=7)$, (C) diabetic C57BL/6 $(\mathrm{n}=9)$, and (D) diabetic Bmp4 $+/-(\mathrm{n}=9)$ mice. These mice were euthanized 24 weeks after the final injection of STZ and citrate buffer. Glycoalbumin levels were measured using an enzyme assay (Lucica GA-L, Asahi Kasei Co., Ltd.). Microalbuminuria was measured using a DCA vantage analyzer (SIEMENS Healthineers). The creatinine concentration was measured with an enzyme assay (Serotec Co., Ltd.). The procedure for generating tamoxifen-inducible Bmp $4 \mathrm{tgm}$ is described in a previous report $^{7}$. Eight- to 10 -week-old transgenic mice were fed a diet (CE-2, CLEA Japan) containing 0.02\% tamoxifen citrate (Sigma) to induce the expression of the Bmp4 gene. Control mice were fed a normal diet (CE-2). The $C A G-C A T-B m p 4$ transgenic mice (CAG-B4) were provided by RIKEN Bio Resource Center (Tsukuba, Japan) under an agreement with Dr. Y. Saga (National Institute of Genetics, Japan). We generated the double tgm by mating CAG-B4 mice with Pod-Cre mice. CAG-B4 x Pod-Cre mice overexpressed BMP4 in podocytes. Reference mice were not mated with Pod-Cre mice. These mice were monitored for 1 year. Blood pressure levels were measured with a non-invasive blood pressure device (BP-98A-L, Softron Co., Ltd.) attached to the animal's tail.

Morphology studies. Tissues used for light microscopy studies were fixed with $10 \%$ neutral buffered formalin. One- $\mu \mathrm{m}$-thick sections were prepared from paraffin-embedded tissues and subjected to periodic acid-Schiff (PAS), periodic acid methenamine silver (PAM) and picrosirius red staining. Morphometric analyses of PAM-stained tissues were performed using ImageJ software. The glomerular surface area and the PAM-positive area/glomerular area (\%) were measured for 30 glomeruli in each mouse.

The avidin-biotin complex ( $\mathrm{ABC}$ ) method was employed for immunohistochemistry to detect the expression of various proteins. Tissue sections were deparaffinized, rehydrated with PBS and treated with $3 \% \mathrm{H}_{2} \mathrm{O}_{2}$. Subsequently, tissue sections were subjected to an antigen retrieval procedure in HistoVT one buffer according to the manufacturer's instructions (Nacalai Tesque, Inc., Japan). Sections were incubated with various primary antibodies. Briefly, 4 - $\mu \mathrm{m}$-thick cryostat sections were prepared, air-dried for an hour and fixed with $4 \%$ paraformaldehyde for $20 \mathrm{~min}$ at $4^{\circ} \mathrm{C}$. After equilibrating the sections with PBS, they were incubated with various primary antibodies overnight at $4^{\circ} \mathrm{C}$, washed with PBS and then incubated with FITC-conjugated secondary antibodies conjugated (Thermo Fisher Scientific) for $1 \mathrm{hr}$. Following a PBS wash, tissue sections were covered with a drop of buffered glycerol, mounted with a cover slip and examined with a fluorescence microscope (KEYENCE CORPORATION). Anti-BMP4 (Abcam), anti-WT1 (Santa Cruz Biotechnology), anti-nephrin (IBL), anti-cleaved caspase-3 (Cell Signaling Technology) antibodies were used for these procedures. Nephrin immunoreactivity was quantified by calculating the nephrin-positive areas in the glomeruli using ImageJ software. The WT1-positive cells were counted in the glomeruli.

Tissues used for electron microscopy were fixed with $2.5 \%$ glutaraldehyde. We entrusted electron microscopy analysis to a specialized company (BML, Inc.).

Cell culture experiment. Conditionally immortalized murine podocytes were obtained from a cell line service (Eppelheim, Germany). Podocytes were cultured in RPMI-1640 medium (Sigma) containing 10\% fetal calf serum, $100 \mathrm{U} / \mathrm{ml}$ penicillin, $100 \mathrm{mg} / \mathrm{ml}$ streptomycin (Invitrogen) and $2 \mathrm{mM} \mathrm{L}$-glutamine at $33^{\circ} \mathrm{C}$ in a $5 \% \mathrm{CO}_{2}$ atmosphere. Cells were cultured on type I collagen-coated dishes at $37^{\circ} \mathrm{C}$ for 1 week to induce differentiation. 
A
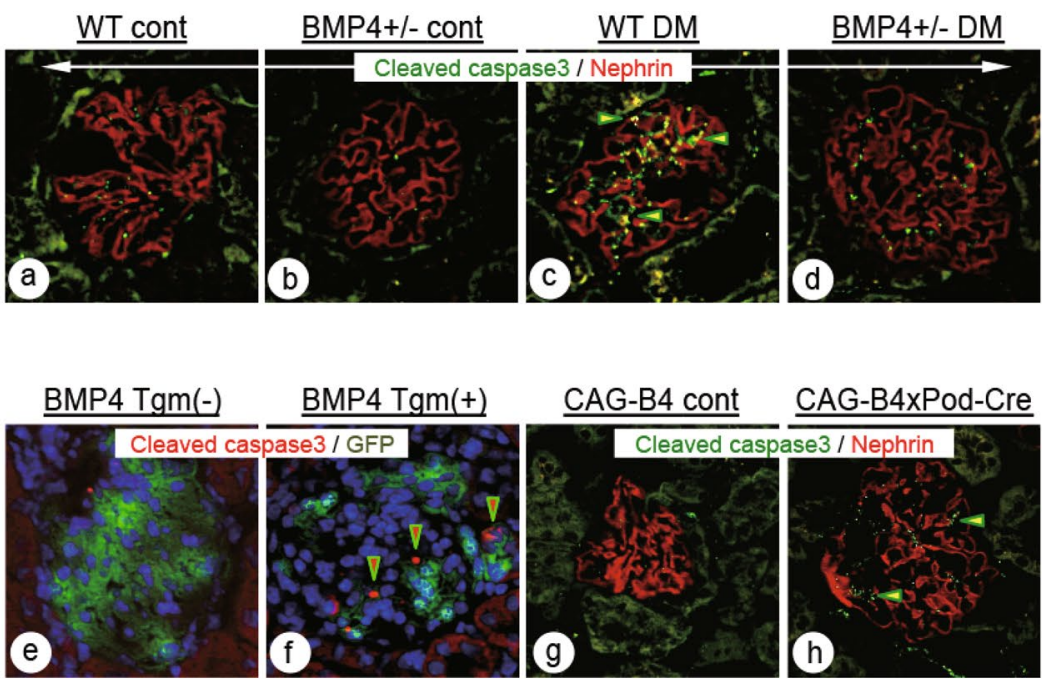

B
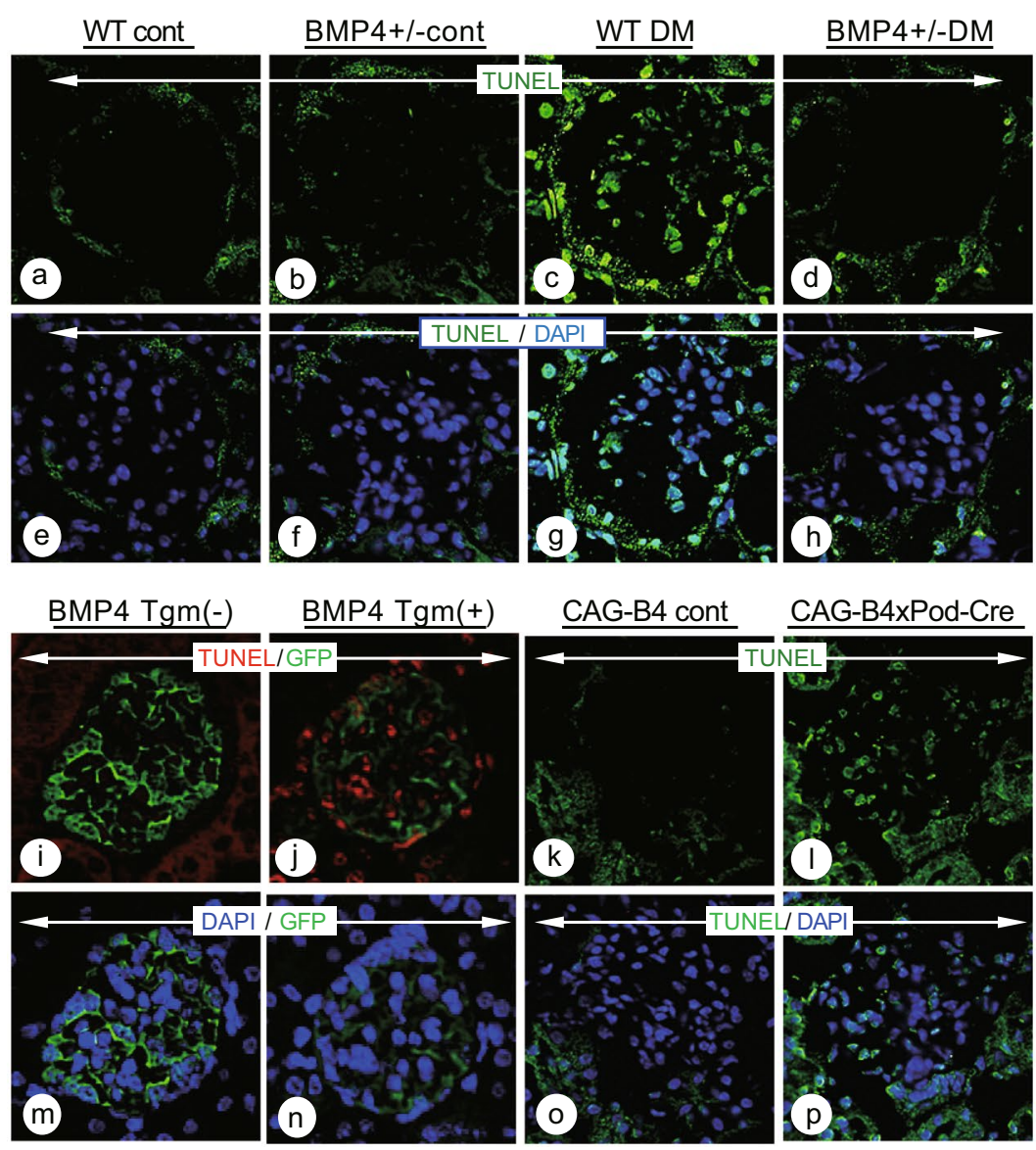

Figure 6. Diabetic mice and BMP4 induced mice exhibited apoptotic damage in glomeruli. (A) Images of immunohistochemistry for cleaved caspase-3 in the DM model, Bmp4 tgm and CAG-B4xPod-Cre mice. The WT DM mice (c) exhibited an increase in the positively stained area compared with the WT control mice (a). Yellow represents the area in which cleaved caspase- 3 and nephrin expression overlap (c, arrowhead). Bmp4 +l-DM mice (d) exhibited a reduced positively stained area compared with WT DM mice. Bmp4 tgm (+) (f) and CAG$B 4 x P o d-C r e(h)$ mice displayed increased positively stained areas after Cre-mediated recombination. Cleaved caspase-3-stained areas are shown in red in Bmp4 tgm $(+)(\mathrm{f}$, arrowhead) and green in CAG-B4xPod-Cre mice (h, arrowhead). (B) Percentage of apoptotic cells in the glomeruli, as determined by a TUNEL assay. Apoptosis was frequently observed in the glomeruli of WT DM (c),Bmp4tgm $(+)(\mathrm{j}$, red color) and CAG-B4xPod-Cre mice (l). Bmp4 $+/-$ DM mice (d) exhibited reduced apoptosis. TUNEL staining is shown in green and red; blue represents the counterstain. 
After differentiation, cells were starved for $24 \mathrm{~h}$ in Opti-MEM (Invitrogen), and then incubated with BMP4 (R\&D Systems) at $37^{\circ} \mathrm{C}$ for the indicated time points. For the inhibition assay, cells were treated with dorsomorphin (Smad1 inhibitor), SB202190, SB242235 or SB203580 (p38 inhibitors) and stimulated with BMP4.

Immunoblot analyses. Cell extracts were prepared from podocytes, as previously described. Protein concentrations in the extracts were measured using the Bradford method, and equal amounts of protein were loaded in each lane of gels, subjected to SDS-PAGE and blotted onto PVDF membranes, which were incubated with a $1 \mu \mathrm{g} / \mathrm{ml}$ dilution of each of the primary antibodies overnight at $4{ }^{\circ} \mathrm{C}$. After a brief wash with TBS-T, membranes were then incubated with a diluted HRP-conjugated secondary antibody (Thermo Fisher Scientific). Autoradiograms were prepared using an enhanced chemiluminescence (ECL) detection system (Thermo Fisher Scientific). Equal loading of the samples was confirmed by probing the immunoblots with $\beta$-actin, $\alpha$-tubulin or GAPDH antibodies. Anti-pSmad1/5/9 (Cell Signaling Technology), anti-pp38 (Cell Signaling Technology), anti-cleaved caspase- 3 (Cell Signaling Technology), anti-podocin (IBL), anti- $\beta$-actin (Sigma), anti- $\alpha$-tubulin (SIGMA), and anti-GAPDH (Abcam) antibodies were used for these procedures. A TUNEL assay was used to detect apoptosis (Millipore).

Statistical analyses. The results of the statistical analyses are reported as the means \pm S.E. Student's t-test or ANOVA was used to compare the data between each group. Albuminuria was statistically analyzed using a nonparametric method. GraphPad Prism 7 software was used for all statistical analyses. $P$ values of less than 0.05 were considered statistically significant.

Availability of data and materials. The tamoxifen-inducible $B m p 4$ transgenic mice are unavailable to the readers because of the licensing agreement between Chugai Pharmaceutical Co., Ltd. and the third party regarding a gene introduced to the mice.

\section{References}

1. Kriz, W., Shirato, I., Nagata, M., LeHir, M. \& Lemley, K. V. The podocyte's response to stress: the enigma of foot process effacement. Am J Physiol Renal Physiol 304, F333-347, https://doi.org/10.1152/ajprenal.00478.2012 (2013).

2. Nagata, M. \& Kriz, W. Glomerular damage after uninephrectomy in young rats. II. Mechanical stress on podocytes as a pathway to sclerosis. Kidney Int 42, 148-160 (1992).

3. Kanwar, Y. S. et al. Diabetic nephropathy: mechanisms of renal disease progression. Exp Biol Med (Maywood) 233, 4-11, https://doi. org/10.3181/0705-MR-134 (2008)

4. Doi, T. et al. Glomerulosclerosis in mice transgenic for growth hormone. Increased mesangial extracellular matrix is correlated with kidney mRNA levels. J Exp Med 173, 1287-1290 (1991).

5. Nagai, K. et al. Mesangial Cell Mammalian Target of Rapamycin Complex 1 Activation Results in Mesangial Expansion. J Am Soc Nephrol, https://doi.org/10.1681/ASN.2016111196 (2017).

6. Abe, H. et al. Type IV collagen is transcriptionally regulated by Smad1 under advanced glycation end product (AGE) stimulation. The Journal of biological chemistry 279, 14201-14206, https://doi.org/10.1074/jbc.M310427200 (2004).

7. Tominaga, T. et al. Activation of bone morphogenetic protein 4 signaling leads to glomerulosclerosis that mimics diabetic nephropathy. The Journal of biological chemistry 286, 20109-20116, https://doi.org/10.1074/jbc.M110.179382 (2011).

8. Mundel, P. \& Shankland, S. J. Podocyte biology and response to injury. J Am Soc Nephrol 13, 3005-3015 (2002).

9. Kriz, W., Gretz, N. \& Lemley, K. V. Progression of glomerular diseases: is the podocyte the culprit? Kidney Int 54, 687-697, https:// doi.org/10.1046/j.1523-1755.1998.00044.x (1998).

10. Wiggins, R. C. The spectrum of podocytopathies: a unifying view of glomerular diseases. Kidney Int 71, 1205-1214, https://doi. org/10.1038/sj.ki.5002222 (2007).

11. D’Agati, V. D., Kaskel, F. J. \& Falk, R. J. Focal segmental glomerulosclerosis. N Engl J Med 365, 2398-2411, https://doi.org/10.1056/ NEJMra1106556 (2011)

12. Asanuma, K. et al. MAP-LC3, a promising autophagosomal marker, is processed during the differentiation and recovery of podocytes from PAN nephrosis. FASEB J 17, 1165-1167, https://doi.org/10.1096/fj.02-0580fje (2003).

13. Isermann, B. et al. Activated protein C protects against diabetic nephropathy by inhibiting endothelial and podocyte apoptosis. Nat Med 13, 1349-1358, https://doi.org/10.1038/nm1667 (2007).

14. Schiffer, M. et al. Apoptosis in podocytes induced by TGF-beta and Smad7. The Journal of clinical investigation 108, 807-816, https:// doi.org/10.1172/JCI12367 (2001).

15. Faure, S., de Santa Barbara, P., Roberts, D. J. \& Whitman, M. Endogenous patterns of BMP signaling during early chick development. Dev Biol 244, 44-65, https://doi.org/10.1006/dbio.2002.0579 (2002).

16. Winnier, G., Blessing, M., Labosky, P. A. \& Hogan, B. L. Bone morphogenetic protein-4 is required for mesoderm formation and patterning in the mouse. Genes Dev 9, 2105-2116 (1995).

17. Michos, O. et al. Reduction of BMP4 activity by gremlin 1 enables ureteric bud outgrowth and GDNF/WNT11 feedback signalling during kidney branching morphogenesis. Development 134, 2397-2405, https://doi.org/10.1242/dev.02861 (2007).

18. Yamaguchi, K. et al. XIAP, a cellular member of the inhibitor of apoptosis protein family, links the receptors to TAB1-TAK1 in the BMP signaling pathway. EMBO J 18, 179-187, https://doi.org/10.1093/emboj/18.1.179 (1999).

19. Hayashi, Y. et al. BMP-SMAD-ID promotes reprogramming to pluripotency by inhibiting p16/INK4A-dependent senescence. Proc Natl Acad Sci USA 113, 13057-13062, https://doi.org/10.1073/pnas.1603668113 (2016).

20. Su, D. et al. BMP4-Smad signaling pathway mediates adriamycin-induced premature senescence in lung cancer cells. The Journal of biological chemistry 284, 12153-12164, https://doi.org/10.1074/jbc.M807930200 (2009).

21. Matsubara, T. et al. Bone Morphogenetic Protein 4 and Smad1 Mediate Extracellular Matrix Production in the Development of Diabetic Nephropathy. Diabetes 64, 2978-2990, https://doi.org/10.2337/db14-0893 (2015).

22. Wang, X., Harris, R. E., Bayston, L. J. \& Ashe, H. L. Type IV collagens regulate BMP signalling in Drosophila. Nature 455, 72-77, https://doi.org/10.1038/nature07214 (2008).

23. Su, D. et al. Role of p38 MAPK pathway in BMP4-mediated Smad-dependent premature senescence in lung cancer cells. Biochem J 433, 333-343, https://doi.org/10.1042/BJ20100404 (2011).

24. Gambaro, K., Aberdam, E., Virolle, T., Aberdam, D. \& Rouleau, M. BMP-4 induces a Smad-dependent apoptotic cell death of mouse embryonic stem cell-derived neural precursors. Cell Death Differ 13, 1075-1087, https://doi.org/10.1038/sj.cdd.4401799 (2006)

25. Lien, S. C. et al. Mechanical regulation of cancer cell apoptosis and autophagy: roles of bone morphogenetic protein receptor, Smad1/5, and p38 MAPK. Biochim Biophys Acta 1833, 3124-3133, https://doi.org/10.1016/j.bbamcr.2013.08.023 (2013). 
26. Kendall, S. E. et al. NRAGE mediates p38 activation and neural progenitor apoptosis via the bone morphogenetic protein signaling cascade. Mol Cell Biol 25, 7711-7724, https://doi.org/10.1128/MCB.25.17.7711-7724.2005 (2005).

27. Pardali, K. et al. Role of Smad proteins and transcription factor Sp1 inp21(Waf1/Cip1) regulation by transforming growth factorbeta. The Journal of biological chemistry 275, 29244-29256, https://doi.org/10.1074/jbc.M909467199 (2000).

28. Arif, E. \& Nihalani, D. Glomerular Filtration Barrier Assembly: An insight. Postdoc J 1, 33-45 (2013).

29. Harvey, S. J. et al. Disruption of glomerular basement membrane charge through podocyte-specific mutation of agrin does not alter glomerular permselectivity. Am J Pathol 171, 139-152, https://doi.org/10.2353/ajpath.2007.061116 (2007).

30. Kulessa, H. \& Hogan, B. L. Generation of a loxP flanked bmp4loxP-lacZ allele marked by conditional lacZ expression. Genesis 32, 66-68 (2002)

\section{Acknowledgements}

We thank Leann B (Springer Nature, U.S.A) for editing the English of the manuscript. We also thank Arisa Ochi, Akiko Sakurai, Sanae Hayashi and Yuriko Morikawa (Tokushima University) for providing technical assistance. We thank the Chugai Research Institute for Medical Science, Inc., as well as our colleagues Chisato Goto for performing animal experiments using the tamoxifen-inducible Bmp4 transgenic mice, Yosuke Kawase, Hiromi Tateishi, Takanori Tachibe and Mami Kakefuda for breeding the tamoxifen-inducible Bmp4 transgenic mice, Toshio Mori for providing technical support for the pathological examinations, and Satomi Uchida for providing excellent technical assistance. We are grateful to Hiroki Kokubo (Hiroshima University), Brigid Hogan (Duke University) and Mitinori Saitou (Kyoto University) for providing the transgenic mice. This study was supported by Grants-in-Aid for Young Scientists (B) (24790848), Grants-in-Aid for Scientific Research (C) (17K09700), an NIH grant (DK60635), and Takeda Science Foundation and Manpei Suzuki Diabetes Foundation funding.

\section{Author Contributions}

Y.F. and T.T. contributed to data analysis and writing of the manuscript. A.H., K.N. and Y.S. contributed to establishing the transgenic mice. K.J., O.U., and N.F. contributed to establishing the transgenic mice and to the experiments. Y.K., S.K. and T.M. contributed to data analysis. Y.S.K. and T.D. guided the investigation and edited the manuscript. T.T., Y.F., and T.D. are the guarantors of this work and, as such, had full access to all study data and take responsibility for the integrity of the data and the accuracy of the data analysis.

\section{Additional Information}

Supplementary information accompanies this paper at https://doi.org/10.1038/s41598-018-31464-9.

Competing Interests: The authors declare no competing interests.

Publisher's note: Springer Nature remains neutral with regard to jurisdictional claims in published maps and institutional affiliations.

(c) (i) Open Access This article is licensed under a Creative Commons Attribution 4.0 International

License, which permits use, sharing, adaptation, distribution and reproduction in any medium or format, as long as you give appropriate credit to the original author(s) and the source, provide a link to the Creative Commons license, and indicate if changes were made. The images or other third party material in this article are included in the article's Creative Commons license, unless indicated otherwise in a credit line to the material. If material is not included in the article's Creative Commons license and your intended use is not permitted by statutory regulation or exceeds the permitted use, you will need to obtain permission directly from the copyright holder. To view a copy of this license, visit http://creativecommons.org/licenses/by/4.0/.

(C) The Author(s) 2018 